\title{
The Impact of Paediatric Anaesthesia Information Leaflet in Reducing Parental and Child Anxiety in Paediatric Surgery
}

Marni Mahfuzah Abdul Wahid

Department of Family Medicine, International Islamic University Malaysia.

Introduction: The process of anaesthesia is one of the most important anxiety precipitating factors for both parent and child who is going for operation. Parental anxiety may adversely affect the children during perioperative period. Studies have shown that anxious parents would want to be provided with more information prior to their child's surgery. We examined the effects of additional paediatric information leaflet on parents and children in reducing pre-operation anxiety. Materials and methods: This was a randomized controlled trial involving 70 parentchild dyads in Hospital Raja Perempuan Zainab II (HRPZ II), Kelantan. Inclusion criteria were child aged 3 to 12 years old who underwent elective surgery. Parents were assigned to intervention and control groups. The former received the information leaflet and the later received standard preparation.. Parents were compared on their anxiety level using Depression Anxiety Stress Scales 21 (DASS-21) (anxiety domain) in the holding area. Children were compared on their emotional state level before induction of anaesthesia using Children's Emotional Manifestation Scale (CEMS). Results: The number of parents who were anxious was similar in both groups $(11(31 \%)$ vs. $7(21 \%), p=.34)$. There were no differences in the children's anxiety state between interventional and control groups as depicted by the CEMS score $(7.5(6.0-9.75)$ vs. $8.0(7.0-15.0), p=.12)$. Conclusion: Parent-child dyads in HRPZ II did not show improvement in the anxiety level with the inclusion of paediatric anaesthesia information leaflet. Further study is needed with larger number of participants involving other selected tertiary hospitals in Malaysia. 